\title{
How to develop new approaches to RFM segmentation
}

Received (in revised form): 24th May, 2004

\section{Amoy X. Yang}

is currently working for General Motors as a senior marketing research consultant at OnStar Division. With an extensive background in electronic engineering, economics and database marketing, his expertise lies in utilising statistical and modelling skills to interpret data for leveraging competitive advantage in decision making. He has obtained several major prizes in his academic field and published numerous articles in influential journals and magazines.

\begin{abstract}
Recency, frequency and monetary (RFM) is a simple and actionable way that has long driven direct marketing efforts. In many cases, however, this empirical segmentation is encumbered with basic shortcomings evident in two aspects: first, the advantage of simplicity often disappears in terms of statistical significance. Secondly, the three-dimensional measure is less predictive than sophisticated models, such as Chi-Square Automatic Interaction Detection (CHAID) and regression analysis. Using RFM as an entry point, this paper discusses the necessity and reality of upgrading this crude method to advanced approaches, where two options are hereby proposed:

- option 1: substituting result-based statistical findings for the traditional intuition-based coding, RFM implementation becomes simple and robust. The transition also advances empirical RFM to CHAID analysis;

- option 2: introducing ' $\mathrm{V}=\mathrm{M} / \mathrm{R}$ ' defined as a customer value, traditional $\mathrm{RFM}$ schemes are readily migrated to individual V-scores without in-depth statistic proceedings. The innovation is compatible to logistic regression.
\end{abstract}

Amoy X. Yang

OnStar Division, General Motors Corporation, Mail Code: 482-D33-C32, 400 Renaissance Center, PO Box 400, Detroit, MI 48265-4000, USA.

Tel: +1313 667 0785; Fax: +1313667 0836; e-mail: amoy_y@yahoo.com

\section{BACKGROUND}

RFM improves targeting by examining when (recency), how often (frequency) and in what purchase amount (monetary) purchase are made. Usually, the more recent, more frequent and higher-dollarvolume customers are more likely to respond to future promotions and, thus, warrant targeting. With this logical rationale, RFM has been widely used and proven as a tried-and-true analysis with which almost every direct marketer begins. ${ }^{1}$

Although the RFM approach has been around for decades, it has not advanced appreciably. ${ }^{2,3}$ Studies from experienced users even suggest eliminating such a crude model. ${ }^{4}$ To reveal the shortcomings attached to this flexible tool, market segmentation basics are re-examined. A true segment means that the customers in each segment must respond differently. ${ }^{5}$ According to this definition, RFM, coded intuitively in terms of behaviours, does not clear up a true segment as follows:

- behavioural difference versus responsive difference: the former provides inferred insights while the latter directly resembles future outcomes. In addition, a customer's 
behaviour is an ordinal type of data that puts forward no quantitative benchmark for true segments; ${ }^{6}$

- visual difference versus statistical difference: how to comprehend the 'difference' is always the central issue from research to practice. Intuitive RFM selection hierarchy states the nominal difference instead of the real difference.

\section{Simplicity against efficiency}

While RFM make apparently common sense as far as marketing and sales can understand, this appealing concept does not favour implementation efficiency. ${ }^{7}$ In other words, an intuitive coding often fails to generate the real differences among RFM cells, which causes a segment-overlapped syndrome. Although some experienced practitioners may flexibly arrange coding categories to pursue what they believe the 'best' differentiations, a subjective approach can never essentially remove the duplication. An overlapping syndrome reduces the RFM modelling efficiency and wastes organisational resources.

The RFM model has continuously suffered from this ever-lasting bottleneck. Historically, the shortcomings of modelling inefficiency are often overlooked, whereas the advantage of simplicity seems over-emphasised.

\section{RFM versus advanced models}

As mentioned before, RFM relies on behavioural similarities to infer the future likelihood of outcome such as response rate (Reps\%). For instance, one can simply anticipate that customers with five purchases should outperform those with four. The true difference between two segments is, however, never warranted. This explains why RFM is deemed to be inferior to CHAID or regression analysis since a crude model counts on intuitive perception while advanced methods benefit from a statistical framework. ${ }^{8}$

With CHAID analysis, the customers' behavioural data and its interaction are interpreted by performance (eg Reps\%). Consequently, Reps\% without real differences can be statistically identified and combined. This procedure eliminates an overlapping syndrome and thus ensures the true market segments. ${ }^{9,10}$

Although regression analysis has no provision to look for interactions automatically, the programmed routine only takes predictors adhering to a pre-established significant level. During the data preparation for multivariate analysis, variables with similar effects on response are aggregated into workable forms that ultimately leverage modelling predictability. ${ }^{11}$

\section{Endeavours on RFM}

RFM is less predictive because the three-dimensional regime is isolated from other data resources (eg demographics). As a traditional solution, new variables (eg gender, age, etc) are simply thrown into RFM to strive for more descriptive clusters such as RFMG, RFMGA, etc. Few studies have yet detailed the procedures because practitioners frequently encounter much difficulty managing the increasing number of cells in reality. ${ }^{12,13}$ The fact is, the unsolved overlapping syndrome significantly holds back this endeavour.

What should be done? If RFM segmentation is to be substantially enhanced to compete with advanced models, the following two options are suggested:

- using the cluster-based approach, the behavioural segments can be restructured in terms of performance that addresses the true segments. This 
Table 1: Hard coding with exact values

\begin{tabular}{llllll}
\hline Coding & $\mathbf{1}$ & $\mathbf{2}$ & $\mathbf{3}$ & $\mathbf{4}$ & $\mathbf{5}$ \\
\hline $\mathrm{R}$ (month) & $19+$ & $13-18$ & $8-12$ & $4-7$ & $1-3$ \\
$\mathrm{~F}$ (times) & 1 & $2-3$ & $4-6$ & $7-10$ & $11+$ \\
M (spending) & $\$ 0-19.99$ & $\$ 20-49.99$ & $\$ 50-99.99$ & $\$ 100-199.99$ & $\$ 200+$ \\
\hline
\end{tabular}

effort first solves overlapping afflictions with a handy $z$-score determination and, secondly, bridges the gap between crude RFM and advanced CHAID;

- introducing a single predictor, ' $\mathrm{V}=\mathrm{M} / \mathrm{R}$ ', which is consolidated from three variables of RFM. The attempt migrates a clustering method to an individual model that is superior to three-dimensional RFM and complementary to a logistic regression.

\section{TRADITIONAL CODING BARRIERS}

Two prevalent forms of coding RFM cells are 'hard-coding' and 'size-coding'. ${ }^{14}$ The former is clustered on the exact values and the latter is enhanced for balancing sizes among cells. Regardless of procedures, both methodologies represent the behavioural segmentation, rather than outcome-based differentiations.

With 'hard coding', the categories in RFM are divided by exact values. As shown in Table 1, the most preferred recency $\left(R_{5}\right)$ falls between ' $1-3$ ' months, the next $\left(\mathrm{R}_{4}\right)$ between '7-12', ... and so forth till the least valuable $\mathrm{R}_{1}$ for 19 months or longer. Similarly, F and $M$ can also be classified by total number of purchases and accumulated spending amount. Linking three variables along with different categories should generate more predictive RFM clusters. If descending codes are assigned to reflect downward performance, $\mathrm{R}_{5} \mathrm{~F}_{5} \mathrm{M}_{5}$ is accordingly ranked at top and $\mathrm{R}_{1} \mathrm{~F}_{1} \mathrm{M}_{1}$ at bottom while others (eg $\mathrm{R}_{3} \mathrm{~F}_{4} \mathrm{M}_{2}$ ) fall in between. The 'hard coding' causes the following problems:

\section{Unidentified interactions}

The function of $\mathrm{R}, \mathrm{F}$ and $\mathrm{M}$ could be overlapped when three variables are simultaneously plugged into the RFM cluster without any data processing. One point worth making is that with 'hard coding' a few variables can produce a notable 'multiplier effect'. Taking the example in Table 1, total RFM cells actually come up with $125(5 \times 5 \times 5)$ that originated from three variables where each includes five categories. Referring to the statistical sampling size to claim the true difference between new finding and mean, say, 5.00 per cent, for instance, 7,300 names are the minimum to achieve 95 per cent confidence within $+/-10$ per cent error range. ${ }^{15,16}$ As a whole, almost 1 million names $(125 \times 7,300)$ are required to fulfil this costly segmentation. As will be discussed later, one cannot but invest a vast amount for duplicated segments. This is obviously undesirable in segmentation efficiency. Hence, data reduction is the first step to fine tune RFM segmentation.

\section{RFM: pros and cons}

RFM coding is fairly arbitrary, which has both pros and cons. A good thing is that the empirical method seems always workable with a flexible coding system. ${ }^{17}$ This implies that RFM should not be abandoned but employed as a crucial predictor to boost the power of market 
Table 2: Data reduction through factor analysis

\begin{tabular}{lllllll}
\hline Simple relationship & Reps\% R & Reps\% F & Reps\% M & R F & R M & F M \\
\hline Correlation $-r$ & 0.89 & 0.41 & 0.37 & 0.19 & 0.21 & 0.93 \\
Strength of relationship & Strong & Weak & Weak & Weak & Weak & Strong \\
Variables included & R & n/a & n/a & n/a & n/a & F or M \\
\hline
\end{tabular}

segmentations. ${ }^{18}$ On the flip side, RFM, coded mostly with experience, may largely skew the true segments and result in an overlapping syndrome. Cutting cells with fewer categories certainly helps ease the complexity. For lack of a statistical framework, one may risk losing significant segments while retaining insignificant ones.

In addition, the 'hard coding' process generates uneven sizes that further threaten segmentation efficiency. For example, smaller cells do not meet statistical significance while larger ones waste extra inputs. By averaging coding cells, the 'size-code' proceeding balances those unequal sizes but does not alleviate overlapping problems.

\section{OPTION 1: RFM CODING RESTRUCTURE}

As concluded above, the assumption of RFM simplicity no longer holds true in terms of statistical reliability. Although coding flexibilities help reduce the complexity, a subjective view cannot sustain statistical precision. To settle this issue, the traditional intuition-based approach must be replaced in order to build a new RFM system that is simple and robust. There are several stages to complete this task.

\section{Stage 1: Data reduction for RFM}

All highly-correlated variables should be integrated into a workable composite that makes the model not only simple but also reliable. ${ }^{19,20}$ In this technical sense, it is necessary to first examine how
$\mathrm{R}, \mathrm{F}$ and $\mathrm{M}$ interact with each other and how they affect Reps\% respectively. As depicted in Table 2, ' $\mathrm{R}$ ' is paramount in driving Reps\% with a correlation of 0.89 , which is probably true in most direct marketing situations. ${ }^{21,22} \mathrm{~F}$ and $\mathrm{M}$ can be factorised into a composite due to their strong correlation $(r=0.93)$. It is a common fact that a larger monetary component is usually accompanied by more frequencies. ${ }^{23}$ To better address revenue and/or profitability, the use of $M$ as a substitute for $F$ is suggested..$^{24}$ As RFM is condensed into RM, the number of cells is significantly reduced from $125(5 \times 5 \times 5)$ to $25(5 \times 5)$.

\section{Stage 2: Coding for $\mathbf{R}$ and $M$}

Starting with the most significant variable (R), Reps\% is then calculated in each month as illustrated in Table 3. How to aggregate $\mathrm{R}$ from each month depends on a statistical $z$-score that claims the true difference between the result-based categories at a desirable confidence level. The formula for calculating $z$ is provided below, together with the commonly used confidences in relation to $z$, as listed in Table $4:^{25,26}$

$$
z=\frac{\left(p_{2}-p_{1}\right)}{\sqrt{p(1-p)\left(\frac{1}{n_{1}}+\frac{1}{n_{2}}\right)}}
$$

where $\mathrm{p}_{1}$ stands for the benchmark (ie Avg. Reps\% or breakeven) based on population $n_{1}$ and $p_{2}$ for Reps $\%$ on cell size $n_{2} \cdot p$ is the weighted average between $p_{1}$ and $p_{2}$. Therefore, $z$ is a parameter that identifies if the real 
Table 3: Result-based coding by $\mathrm{R}$

\begin{tabular}{lccrr}
\hline $\mathbf{R}($ Month) & $\begin{array}{l}\text { Volume of } \\
\text { mail }\end{array}$ & $\begin{array}{l}\text { Number of } \\
\text { responses }\end{array}$ & Reps $\%$ & z-score \\
\hline$R_{3}(1)$ & 1,192 & 117 & 9.82 & 7.54 \\
$R_{3}(2)$ & 3,123 & 334 & 10.69 & 14.15 \\
$R_{3}(3)$ & 2,730 & 258 & 9.45 & 10.41 \\
$R_{3}(4)$ & 3,941 & 335 & 8.50 & 9.77 \\
$R_{3}(5)$ & 7,872 & 540 & 6.86 & 7.20 \\
$R_{3}(6)$ & 4,091 & 324 & 7.92 & 8.31 \\
$R_{3}(7)$ & 5,288 & 329 & 6.22 & 3.95 \\
$R_{3}(8)$ & 6,710 & 375 & 5.59 & 2.13 \\
$R_{2}(9)$ & 4,293 & 214 & 4.98 & -0.04 \\
$R_{2}(10)$ & 3,003 & 157 & 5.23 & 0.56 \\
$R_{2}(11)$ & 938 & 58 & 6.18 & 1.65 \\
$R_{2}(12)$ & 2,795 & 139 & 4.97 & -0.06 \\
$R_{2}(13)$ & 3,810 & 194 & 5.09 & 0.26 \\
$R_{2}(14)$ & 5,206 & 117 & 4.46 & -1.76 \\
$R_{1}(15)$ & 3,093 & 169 & 3.78 & -3.07 \\
$R_{1}(16)$ & 4,064 & $\ldots .16$ & -2.42 \\
$\ldots$ & $\ldots, \ldots 00$ & $\ldots .00$ & $\ldots$ \\
$R_{321}(1-24)$ & 100,000 & & 5.00 \\
\hline
\end{tabular}

difference exists in terms of nominal differences $\left(p_{2}-p_{1}\right)$ adjusted by the sample sizes $\left(n_{1}\right.$ and $\left.n_{2}\right)$. As an example in Table 3, when compared to benchmark of 5.00 per cent at 95 per cent confidence, a higher Reps $\%$ of 6.18 per cent (in 11th month) does not exhibit a real lift due to the smaller sample size of 938, whereas a lower Reps\% of 5.59 per cent (in 8th month) achieves the statistical difference with its larger size of 6,710 . This example shows what appears to be intuitively differentiated might not achieve the reliable results due to the insignificant sample size. Only when the percentage is put in the context of the sample size can the segments be found in terms of statistical framework.

Knowing how to classify the true

Table 4: Test statistic

\begin{tabular}{ll}
\hline z-score & Confidence \% \\
\hline 0.674 & 50 \\
0.842 & 60 \\
1.036 & 70 \\
1.282 & 80 \\
1.645 & 90 \\
1.960 & 95 \\
2.576 & 99 \\
\hline
\end{tabular}

segments, the most efficient way to approach an analytic goal can be utilised. For instance, to maximise the overall profitable responses through RFM segmentation, all that needs to be done is profiling the customers in three categories - positive, neutral and negative in comparison to breakeven point. Presumably, a breakeven rate (BE per cent) equates to 5.00 per cent $(5,000 / 100,000)$, that is the same as an average Reps\%. With the benchmark of 5.00 per cent at a 95 per cent confidence level, Reps\% is calculated for each monthly $\mathrm{R}$ in Table 3 , and then the similar results are aggregated into the following three distinct schemes (as found in Table 5).

- $\mathrm{R}_{3}(1-8)$ shows the real lift by lumping the categories where $z>=1.96$;

- $\mathrm{R}_{2}(9-14)$ is neutral when $z$ falls between -1.96 and 1.96 ;

- $\mathrm{R}_{1}(15+)$ significantly runs below benchmark if $z<=-1.96$.

Thus, a result-based coding translates five blurred R-categories (as shown in Table 1) into three true segments. Under 
Table 5: Result-based coding by $\mathrm{R}$

\begin{tabular}{lcccr}
\hline Recency & $\begin{array}{l}\text { Volume of } \\
\text { mail }\end{array}$ & $\begin{array}{l}\text { Number of } \\
\text { responses }\end{array}$ & Reps\% & z-score \\
\hline$R_{3}(1-8)$ & 34,947 & 2,612 & 7.47 & 17.26 \\
$R_{2}(9-14)$ & 20,045 & 994 & 4.96 & -0.24 \\
$R_{1}(15+)$ & 45,008 & 1,394 & 3.10 & -16.33 \\
$R_{3,2,1}$ & 100,000 & 5,000 & 5.00 & 0.00 \\
\hline
\end{tabular}

Table 6: Result-based coding by $M$

\begin{tabular}{lcllr}
\hline Monetary & $\begin{array}{l}\text { Volume of } \\
\text { mail }\end{array}$ & $\begin{array}{l}\text { Number of } \\
\text { responses }\end{array}$ & Reps $\%$ & z-score \\
\hline$M_{3}(\$ 66+)$ & 32,800 & 1,897 & 5.78 & 5.55 \\
$M_{2}(\$ 18-65)$ & 30,700 & 1,556 & 5.07 & 0.48 \\
$M_{1}(\$ 17-)$ & 36,500 & 1,547 & 4.24 & -5.83 \\
$M_{3,2,1}$ & 100,000 & 5,000 & 5.00 & 0.00 \\
\hline
\end{tabular}

Table 7: Result-based coding by $\mathrm{R}_{2} \mathrm{M}_{3}$

\begin{tabular}{lllll}
\hline $\mathbf{R}+\mathbf{M}$ & $\begin{array}{l}\text { Volume of } \\
\text { mail }\end{array}$ & $\begin{array}{l}\text { Number of } \\
\text { responses }\end{array}$ & Reps\% & z-score \\
\hline $\mathrm{R}_{2} \mathrm{M}_{3}$ & 6,225 & 341 & 5.48 & 1.67 \\
\hline
\end{tabular}

similar circumstances, $M_{3}, M_{2}$ and $M_{1}$ are obtained in Table 6 . Since $\mathrm{R}$ indicates larger differentiations than $\mathrm{M}$, $\mathrm{R}$ is entered into the first layer of a segmenting tree diagram. As depicted in Figure $1, \mathrm{R}_{3}$ stands out as the first targeting segment while $R_{2}$ and $R_{1}$ need to be driven by the other powerful vehicle like $\mathrm{M}_{3}$.

When moving to the second layer in Figure 1, the new combination of $\mathrm{R}_{2} \mathrm{M}_{3}$ raises Reps $\%$ with $z$-score at 1.67 that merely hits a 90 per cent confidence. To satisfy a pre-established 95 per cent level, it is necessary to go beyond RFM constraint for further enhancement. The difference here is, instead of dealing with all 125 'hard coding' cells, only one significant cell $\left(\mathrm{R}_{2} \mathrm{M}_{3}\right)$ is worked on for subsegment(s). As such, segmentation efficiency is dramatically improved.

\section{Stage 3: Move beyond pure RFM}

Classification based on statistical findings is the technique used in CHAID. ${ }^{27}$ In this context, result-based RFM can quickly advance to sophisticated analysis for searching finer segments. That is to say, RFM segmentation, if statistically identified, can be directly enhanced by CHAID analysis.

To lift $\mathrm{R}_{2} \mathrm{M}_{3}$, a new variable is recruited - gender, denoted by $G_{1}$ for male and $G_{2}$ for female. Why is gender first embedded instead of the other demographic variables? As a rule of thumb, a new entry is prioritised in terms of 'bigger' lift along with a 'larger' size according to the formula of calculating z-score. $\mathrm{G}_{2}$ meets the criteria by significantly lifting $\mathrm{R}_{2} \mathrm{M}_{3}$ performance as shown in Table 8 . This newly-created cell $\mathrm{R}_{2} \mathrm{M}_{3} \mathrm{G}_{2}$ joins the previous $R_{3}$ for targeting purpose, where both cells are highlighted in Table 9 and mapped in Figure 1.

Can more significant segments be found to this end? Few possibilities exist by looking at $R_{1}, R_{2} M_{2}$ and $R_{2} M_{3} G_{1}$.

- $\mathrm{R}_{1}$ shows an extremely negative $z$-score, which is less likely to be boosted unless an exceptional lifting impact could be found; 
Table 8: Result-based coding by $\mathrm{R}_{2} \mathrm{M}_{3} \mathrm{G}$

\begin{tabular}{lllll}
\hline $\mathbf{R}+\mathbf{M}+\mathbf{G}$ & $\begin{array}{l}\text { Volume of } \\
\text { mail }\end{array}$ & $\begin{array}{l}\text { Number of } \\
\text { responses }\end{array}$ & Reps\% & z-score \\
\hline$R_{2} M_{3} G_{2}$ & 3,217 & 188 & 5.84 & 2.16 \\
$R_{2} M_{3} G_{1}$ & 3,008 & 153 & 5.09 & 0.21 \\
\hline
\end{tabular}

Table 9: Targeting efficiency: OfMail vs. OfReps

\begin{tabular}{lcllrr}
\hline Recency & $\begin{array}{l}\text { Volume of } \\
\text { mail }\end{array}$ & $\begin{array}{l}\text { Number of } \\
\text { responses }\end{array}$ & Reps \% & \% OfMail & \% OfReps \\
\hline$R_{3}$ & 34,947 & 2,612 & 7.47 & 35 & 52 \\
$\mathrm{R}_{2} \mathrm{M}_{3} \mathrm{G}_{2}$ & 3,217 & 188 & 5.84 & 3 & 4 \\
Others & 61,836 & 2,200 & 3.56 & 62 & 44 \\
Sum $/$ Avg & 100,000 & 5,000 & 5.00 & 100 & 100 \\
$\mathrm{R}_{3}+\mathrm{R}_{2} \mathrm{M}_{3} \mathrm{G}_{2}$ & 38,164 & 2,800 & 7.34 & 38 & 56 \\
\hline
\end{tabular}

- $\mathrm{R}_{2} \mathrm{M}_{2}$ has no chance to be significantly positive since the stronger $\mathrm{R}_{2} \mathrm{M}_{3}$ barely catches up to 90 per cent confidence level;

- $\mathrm{R}_{2} \mathrm{M}_{3} \mathrm{G}_{1}$, with a limited sample of 3,008 , provides little room for further statistical findings. ${ }^{28}$

To address the analytic goal, both $\mathrm{R}_{3}$ $(34,924)$ and $\mathrm{R}_{2} \mathrm{M}_{3} \mathrm{G}_{1}(3,217)$ are embraced. It ends up with 38,164 $(34,947+3,217)$, or 38 per cent $(38,164 / 100,000)$ of total leads. As BE\% is equal to average Reps\% of 5.00 per cent, the targeting distribution can be roughly estimated with one half above $\mathrm{BE} \%$ and the other half below $\mathrm{BE} \%$. Thirty eight per cent is a satisfactory portion that has been discovered to approach a maximum profitability since approximately $5-10$ per cent leads cannot be significantly identified. Undoubtedly, RFM dominates the overall segmentation.

Figure 1 outlines a CHAID tree diagram initiated from the foremost $\mathrm{R}$, $M$ and $G$ in sequence. The coding transition from 'intuitive base' to 'resultant base' simplifies the analytical process and provides explicit insights for decision making. Adopting a handy $z$-score formulation, a newly-structured RFM improves predictive precision, realises cost effectiveness and increases odds for new findings.

Finally, overall segmentation efficiency is reviewed. In mass market, 100,000 mails (ie 100 per cent of total contacts) generate 5,000 sales (ie 100 per cent of overall responses), where no profits could be made since an overall

Reps $\%=\mathrm{BE} \%=5.00$ per cent. Through market segmentation, profitable segments $\left(\mathrm{R}_{3}+\mathrm{R}_{2} \mathrm{M}_{3} \mathrm{G}_{2}\right)$ take 38 per cent of all mails but yield 56 per cent of overall responses. As shown in Table 10, the difference by 18 per cent (56 per cent -38 per cent) produces additional responses of 900 ( 18 per cent $\times 5,000)$. An ultimate ROI will be multiply increased while deploying this execution to foregoing promotions.

\section{OPTION 2: INTRODUCE 'V $=\mathbf{M} / \mathbf{R}^{\prime}$}

CHAID can greatly aid RFM. These cluster-based segments, however, are often constrained by inadequate sampling sizes where the situation can be significantly exacerbated by diving into deeper layers. Moreover, customers in 


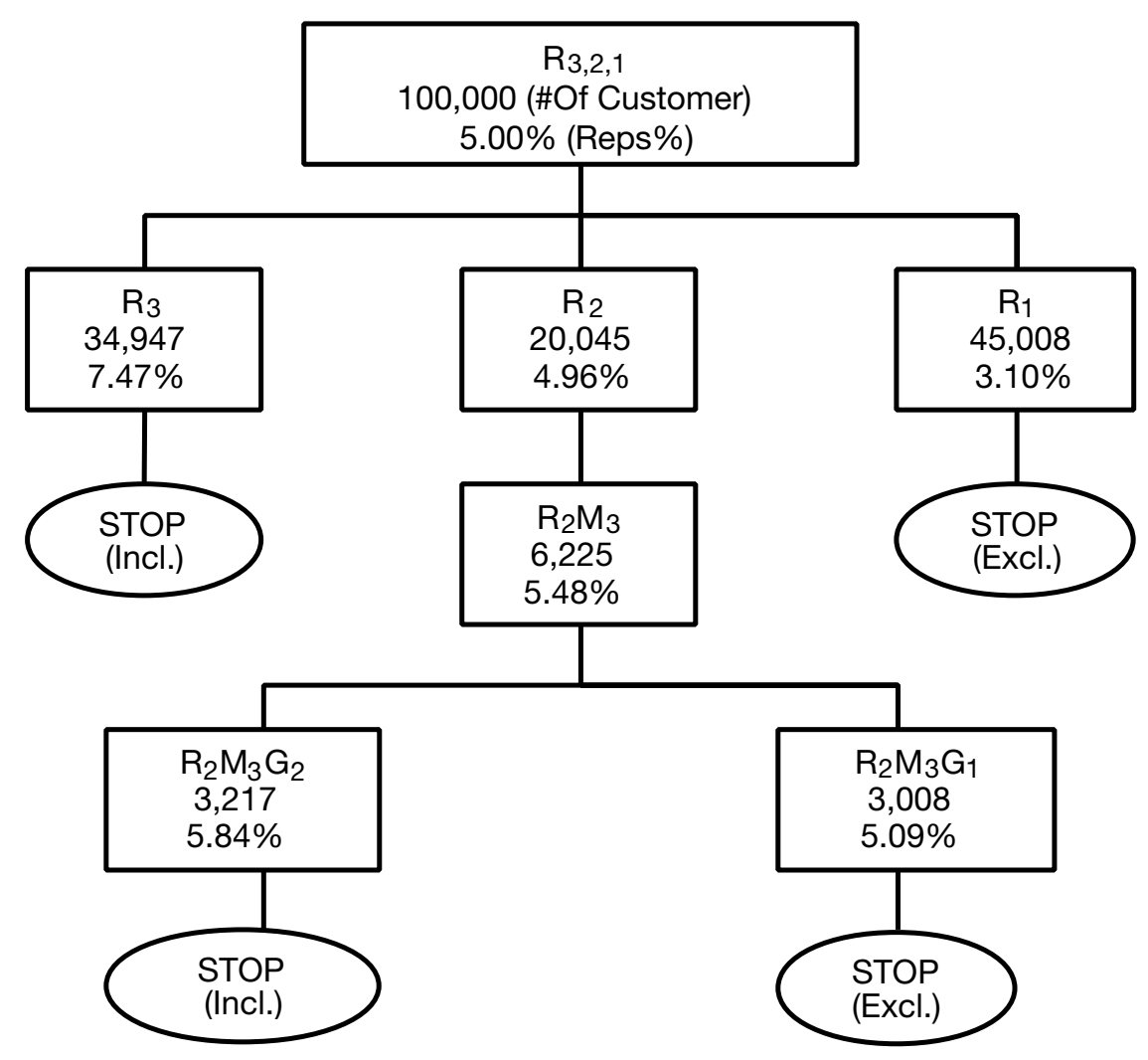

Figure 1 CHAID tree diagram starting with RFM

the same cluster cannot be differentiated. ${ }^{29}$

\section{Individual approach}

Compared to cluster segmentation, an individual model (eg logistic regression) can overcome the difficulties above by probing into every single differentiation within one sampling data file. An advanced approach, however, relies heavily on a statistician familiar with the techniques and sophisticated tools such as SAS or SPSS. This may not be attainable or worthwhile to some practitioners, especially to small organisations. As an alternative, this paper introduces a new term — ' $\mathrm{M} / \mathrm{R}$ ' — simulated from empirical RFM concepts. The next step will detail how to put three categories (RFM) into one single factor that is compatible to a logistic performance.

\section{From RFM to M/R}

As concluded before, RFM has been reduced to $R$ and $M$ through factor analysis. Incorporating $\mathrm{R}$ with $\mathrm{M}$ requires marketing intelligence and quantitative disciplines. The product of $\mathrm{R} \star \mathrm{M}$, measured by 'month ${ }^{\star}$ dollar', is improper to be literally defined. On the other hand, customers ranked by RFM are directly related to $M$, yet reciprocally to $R$. The ratio of ' $M / R$ ' simply reflects these relationships. To be more specific, ' $M / R$ ' values a customer in terms of his/her historic spending amount (M) over the time interval ( $R$ ) between now and the most recent purchase date. Let ' $\mathrm{V}=\mathrm{M} / \mathrm{R}$ ', which stands for customer value favouring larger spending with more recency. Corresponding to the RFM principle, $\mathrm{V}$ represents the past value that infers future responding likelihood. Recall that $\mathrm{R}$ and $\mathrm{M}$ are 
Table 10: Reps\% versus $V$ (subscript $x$ refers to variables indexed at 1 )

\begin{tabular}{|c|c|c|c|c|c|c|}
\hline Reps \% & $\mathbf{R}(\mathbf{Q t})$. & $\mathbf{M}(\$)$ & 1/R & M/R & $($ Reps \%) & $(M / R)_{x}$ \\
\hline 9.50 & 1 & $\$ 64$ & 1.00 & 64.0 & 1.00 & 1.00 \\
\hline 6.54 & 2 & $\$ 68$ & 0.50 & 34.0 & 0.69 & 0.53 \\
\hline 5.15 & 3 & $\$ 76$ & 0.33 & 25.3 & 0.54 & 0.40 \\
\hline 4.40 & 4 & $\$ 62$ & 0.25 & 15.5 & 0.46 & 0.24 \\
\hline 3.63 & 5 & $\$ 56$ & 0.20 & 11.2 & 0.38 & 0.18 \\
\hline 3.46 & 6 & $\$ 59$ & 0.17 & 9.8 & 0.36 & 0.15 \\
\hline 3.11 & 7 & $\$ 61$ & 0.14 & 8.7 & 0.33 & 0.14 \\
\hline 2.41 & 8 & $\$ 45$ & 0.13 & 5.6 & 0.25 & 0.09 \\
\hline \multirow[t]{2}{*}{ Corr-r } & -0.920 & 0.537 & 0.983 & 0.993 & & 0.993 \\
\hline & Reps\% R & Reps\% M & Reps\% 1/R & Reps\% M/R & & $(\operatorname{Reps} \%)_{x} \sim(M / R)_{x}$ \\
\hline
\end{tabular}

intuitively determined during 'hard coding' procedure. ' $\mathrm{M} / \mathrm{R}$ ' provides the baseline from which to work through more technical details to attain a practical model. As an optimal goal, $\mathrm{R}$ and $\mathrm{M}$ should be well coordinated to coincide with the actual responses (ie Reps\%).
... etc. This is the algorithm that adjusts $\mathrm{R}$ with a given $\mathrm{M}$ to fit the actual Reps\%, where $\mathrm{R}$ can be appropriately shortened (eg bimonthly) if it under-paces Reps\% or extended (eg biannual) if it overpasses Reps\%.
- ' $R$ ' accounts for the time period typically on a monthly, quarterly or biannual basis. A shorter time unit implies that $\mathrm{R}$, compared to $\mathrm{M}$, is more critical to $\mathrm{Reps} \%$, and vice versa. To approach the most suitable unit for R, a few trials can explore how $1 / \mathrm{R}$ copes with $\mathrm{Reps} \%$. In reality, $\mathrm{R}$ is typically rounded up to an averaged interval between two consecutive campaigns since later respondents should not be credited more than earlier ones within the same tracking window. Considering a normal tracking period between 60 and 90 days in the direct mail industry, R should be set up on a quarterly incremental base $;^{30}$

— 'M', simply measured by dollar, is straightforward to describe ' $\mathrm{V}=\mathrm{M} / \mathrm{R}$ '. For example, a customer who spends a total of $\$ 50$ with ' $\mathrm{R}=1$ ' has the same $\mathrm{V}$ as another customer who spends $\$ 100$ with $' \mathrm{R}=2$ '. When the proportional multiplier is applied to both $\mathrm{M}$ and $\mathrm{R}$, this gives: $50 / 1=100 / 2=150 / 3$

\section{Validity and efficiency}

To verify how well each individual $\mathrm{V}$ (ie $M / R$ ) imitates the binary response (yes or no), a categorical correlation needs to be conducted by using SAS or SPSS, which is out of scope of this paper. Instead, the author suggests a quick approach that examines cluster-based correlation ( $\mathrm{r}$ ) between Reps\% and $\mathrm{M} / \mathrm{R}$. As such, a larger ' $\mathrm{r}$ ' indicates the better segments that are associated with more accurate individual scores, and vice versa.

As illustrated in Table 10, Reps\% is strongly related to ' $\mathrm{M} / \mathrm{R}$ ' because $r=0.993$. This outcome proves that a single predictor, ' $\mathrm{M} / \mathrm{R}$ ', provides a more flexible and insightful alternative to scoring or segmenting customers than empirical RFM. Then why is the single variable so informative and predictive? ' $1 / \mathrm{R}$ ' significantly improves traditional linearity of ' $R$ ' because the reciprocal function ' $1 / \mathrm{R}$ ' well fits the fast-to-slow declining trend of Reps\%. ${ }^{31}$ As recorded in Table 10, ' $1 / \mathrm{R}$ ' forms a solid 


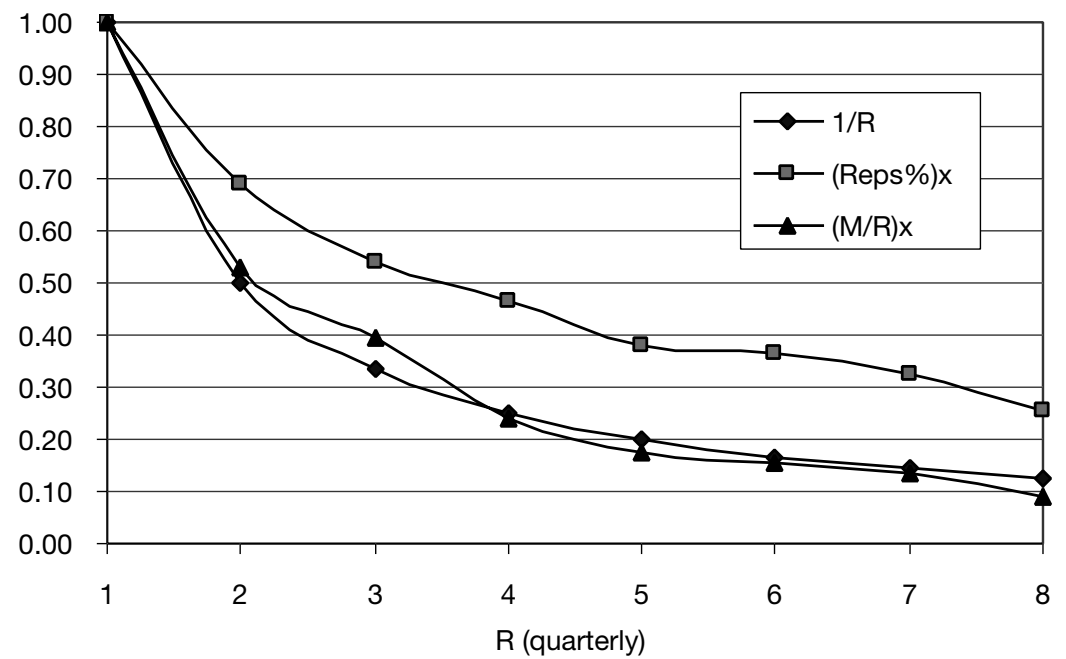

Figure 2 Validation for Reps\% vs 'M/R'

foundation for achieving ' $\mathrm{r}$ ' as high as 0.983. This phenomenon is consistent with the following historic evidences cited in literatures:

— R plays a leading role within a RFM cell; ${ }^{32,33}$

- as an example shown in Table 1, experienced practitioners often code $\mathrm{R}$ from narrower to wider ranges in accordance with this characteristic; ${ }^{34,35}$

- a larger portion is encompassed in the lowest performed cells such as $\mathrm{R}_{1} \mathrm{~F}_{1} \mathrm{M}_{1} \cdot{ }^{36,37}$

Notice the dilemma to draw the relationships among Reps\%, 'M/R' and ' $1 / \mathrm{R}$ ' due to their non-comparable measures. In this regard, each variable's scale must be indexed at the same benchmark, where the base is the ' $1 / \mathrm{R}$ ' (from 1 to 0 ) for adjusting the others. Since $r$ remains the same from non-indexed ' $M / R$ ' to indexed (M/R)x, as found in Table 10, Figure 2 virtually describes the relationship between Reps\% and 'M/R'. As a result, ' $1 / \mathrm{R}$ ' closely traces the curvilinear growth of Reps $\%$. With an auxiliary amendment by ' $M$ ', a refined score — ' $M / R$ ' - is therefore obtained. ${ }^{38}$ Besides, an indexed $(\mathrm{M} / \mathrm{R}) \mathrm{x}$, ranged from 0 to 1 , provides a logical comparison to the probabilities computed from the logistic model.

\section{SUMMARY}

The underlying rationale of empirical RFM is simple and useful, while its implementation could be inefficient due to multiple blurred schemes or an overlapping syndrome. Even though the flexibility inherent in RFM can simplify segmentation, these intuition-coded clusters are incapable of addressing true segments. ${ }^{39}$ The fundamental way out for crude RFM lies in result-based segments that can be truly identified by a statistical ' $z$-score'. With a little quantitative effort, direct marketers can realise tremendous returns that significantly leverage both applicable simplicity and statistical reliability. Additionally, this new approach advances RFM to CHAID, where more sophisticated breakdowns can be revealed.

Further improvement for RFM centres on the transition from a segment-based approach to an individual-scored focus. Introducing ' $\mathrm{V}=\mathrm{M} / \mathrm{R}$ ' based on RFM 
principle, each customer's value $(\mathrm{V})$ can be readily assessed by a ratio of ' $M / R$ '. As it has been validated, the new individual model not only surpasses empirical RFM, but also adds the bottom-line of sophisticated logistic regression.

Finally, the two options proposed above embody the essence of RFM while compensating for the pitfalls incurred in its empirical implementation. Both state-of-the art approaches are vital to today's direct marketing industry.

\section{Acknowledgments}

The author thanks Dr Michael Jennings, campaign analyst of General Motors Corporation, and two anonymous referees for their valuable comments and suggestions.

\section{References}

1 Dicky, C. (2002) 'Develop new approaches to analysis', DM News, 26th August, p. 28.

2 Miglautsch, J. (1995) 'Insight RFM segmentation modeling', DM News, 16th October, p. 22.

3 McAvoy, P. (2003) 'Simple yet sophisticated', Catalog Age, Vol. 20, No. 12, pp. 63-68.

4 Wheaton, J. (1996) 'The superiority of tree analysis over RFM: How it enhances regression', DM News, 12th August, pp. 21-23.

5 Neal, W. D. (2004) 'Principles of marketing segmentation', Marketing Research, April. http://www.marketingpower.com/live/ content1006.php

6 Lewis, B. R. and Ford, R. K. (1987) 'Basic statistics using SAS', 2nd edn, West Publishing Company, Minnesota.

7 Marcus, C. (1998) 'A practical yet meaningful approach to customer segmentation', Journal of Consumer Marketing, Vol. 15, No. 5, pp. 494-502.

8 Wheaton (1996) op. cit.

9 Hansotia, B. J. and Rukstales, B. (2002) 'Direct marketing for multi-channel retailers: Issues, challenges and solutions', Journal of Database Marketing, Vol. 9, No. 3, pp. 259-275.

10 David Shepard Associates, Inc. (1990) 'How to implement a profit-driven database marketing strategy', The New Direct Marketing, Business One Irwin, Illinois.

11 Wheaton, J. (1996) 'RFM cells: The "Kudzu" of segmentation', DM News, 15th July, p. 28.

12 Ibid.

13 Retka, R. (2001) 'Beyond traditional segmentation', DM News, 5th November, p. 30.
14 Hughes, A. M. (1996) 'Why RFM deserves its honored place', DM News, 23rd September, p. 33.

15 Yang, A. X. (2003) 'How to design a valid DM sampling test', Direct Marketing Magazine, October, http://www.directmarketingmag.com/news/ 450146.html

16 Wheaton, J. (2001) 'How big should my test be', DM News, 1st October, pp. 26-27.

17 Dicky (2002) op. cit.

18 Ables, G. (1997) 'Predictive modeling for non-statisticians', Target Marketing, Vol. 20, No. 3, pp. 114-115.

19 Lyons, K. (2001) 'Factor and cluster analysis in CRM', DM News, 21st May, p. 21.

20 Sambandam, R. (2003) 'Cluster analysis gets complicated', Marketing Research, Vol. 15, No. 1, pp. 16-26.

21 Miglautsch (1995) op cit.

22 Quaranta, F. (1998) 'Getting back to the list basics: RFM', DM News, 1st June, pp. 32-34.

23 Marcus (1998) op. cit.

24 Weber, A. (1999) 'Segmentation secrets - Dig deep in your database to uncover 19 secrets to profitable customer segmentation', Target Marketing, September, pp. 58-62.

25 Mason, R. D., Lind, D. A. and Marchal, W. (1999) 'Study guide for use with statistical techniques in business and economics, 10th edn, Irwin McGraw-Hill, New York.

26 Drake, P. D. (2000) 'Did your new marketing test really beat the control?',

http://www.drakebusiness.com/Link_Articles_10.html

27 Hansotia and Rukstales (2002) op. cit.

28 Yang (2003) op. cit.

29 Wedel, M. (2001) 'Is segmentation history?', Marketing Research, Vol. 13, No. 4, pp. 26-29.

30 Weber, A. (1997) 'A simple way to use RFM', Target Marketing, Vol. 20, No, 3, pp. 72-75.

31 Hughes, A. (1998) 'When not to model — For customer communications, RFM provides plenty of information', Target Marketing, July, pp. 32, 34 and 75.

32 Miglautsch (1995) op. cit.

33 Miller, P. (1998) 'RFM, to RMP, to RMPF', Catalog Age, Vol. 15, No. 4, p. 47

34 Quaranta (1998) op. cit.

35 Schmid, J. (1998) 'Catalog creative: The RFMP way’, Target Marketing, July, pp. 38-41.

36 Weber (1999) op. cit

37 Miglautsch, J. (2002) 'Application of RFM principle: What to do with 1-1-1 customers?', Journal of Database Marketing, Vol. 9, No. 4, pp. 319-325.

38 Berry, T. (1998) 'Developing an acquisition modeling strategy', DM News, 20th April, pp. 27 and 30 .

39 Fitzpatrick, M. (2001) 'Statistical analysis for direct marketers — In plain English', Direct Marketing Magazine, August, pp. 54-56. 